\title{
An Efficient Approach to De-noising Mammogram Images
}

\author{
Jayant Rajurkar ${ }^{1}$, Shirish Singnapure ${ }^{2}$ \\ Assistant Professor, Department of Computer Engineering, MIET, Bhandara (MS), India ${ }^{1,2}$
}

\begin{abstract}
The noisy nature of digital mammograms and low contrast of doubtful areas which make medical images denoising a challenging problem. In the field of image processing, different types of denoising algorithm are used to remove the unnecessary noise such as additive white Gaussian noise, salt and pepper noise, impulsive noise, as well used to smoothen the image. In the existing work, a novel gradient histogram preservation (GHP) algorithm was developed to improve the texture which exhibits the reduced quality in recognition of human image experiments. To remove the noise, Discrete Wavelet Transform (DWT) algorithm is used to eliminate the noisiness present in the medical image to preserve the texture structures. Proposed approach will efficiently calculate peak signal noise ratio (PSNR) parameters and make the enhanced image without noise and obtains the quality of image without any loss of data.
\end{abstract}

Keywords: Image Denoising, Discrete Wavelet Transform (DWT), Gaussian Filter, Mammogram image, Peak Signalto-Noise Ratio.

\section{INTRODUCTION}

The different noise such as white Gaussian noise, salt and pepper noise, impulsive noise, etc present in the images may appear as additive or multiplicative components and the main function of denoising process is to remove these noisy components while preserving the important signal as much as possible [1]. Therefore, in the field of the medical image pre-processing, method of de-noising plays a very important role. It is often done before the image is to be analyzed [2]. De-noising is mainly used to remove noise that is present and retains the significant information, regardless of the frequency contents of the signal [3]. During the process of de-noising, much attention is kept on how well the edges are preserved and how much of noise granularity been removed[4]. Thus, the main purpose of image denoising algorithm is to eliminate the unwanted noise level while preserving the important features of an image [5].

The rapid advancement in the field of digital image processing has allowed to enhancement, filtering, or any other types of digital image manipulation that may improve diagnostic interpretation [3], [4]. Digital image format is required in computer-aided diagnosis (CAD) schemes to assist the radiologist in the detection of radiological features that could point to different pathologies [12], [13]. Images in digital format constitute an obligatory requisite toward the implementation of both picture archiving and communications systems (PACS) and teleradiology.Tthe conveniences of such new techniques mainly depends on two parameters of importance, one is spatial and another is grey level resolutions. They must provide a diagnostic accuracy in digital images equivalent to that of conventional films. Both pixel size and pixel depth are factors that critically affect the visibility of small-low contrast objects or signals, which often are relevant information for diagnosis [5]. Therefore, digital image recording systems for medical imaging must provide high spatial resolution and high contrast sensitivity [2].

We focus on the current problem by using several proposed methods by providing the better performance that are compared to the existing problem. There are different types of noises available in the image. Some of them are like salt and pepper noise, Gaussian noise, impulsive noise etc. The main aim of these types of noises has to remove from the image or a set of data that is available in the process [5]. The image used in this denoising methodology is Mammogram images,i.e. brain images has been taken as an input image and different methods are being implemented to enhance the texture, smoothness and visual quality which can be improved and enhanced.

The main properties of a good denoising model are used to remove unwanted noise present in the medical image by preserving their edges. The common approach is that by using a Gaussian filter, the images can be resized and fit the desired outcome and can solves the different varieties of equations in the mathematical skill. The main advantage of using noise removal models is quite high in their speed compared to other techniques. The main drawback of this model is, it does not preserve the edges which are resized in the previous model, and was characterized based on their dissimilarities[20]. 
Consequently, de-noising plays a very important role in the field of the medical image pre-processing. It is often done before the image is to be analyzed and thus preprocessing plays an imperative role in image classification task. In general, noise filters can be divided into two types, linear methods and non-linear filtering methods. Linear methods involved methods like median, Weiner, adaptive and mean filters. Non -linear filtering methods includes filters from wavelet domain such as $\mathrm{db} 3, \mathrm{db} 4$ and sym6 ect.

By using various models, we can easily handle the edges as well as the textures in a better manner. The most important methodology used was nonlinear image denoising is the Median filter, which is used to preserve the edges, and replaces the current pixel by the mid of the pixel range and varies with their texture which is based on their input image which is a time domain and transformed into regions as frequency domain as an output image. By developing many denoising algorithm, it reduces the noise in the image and brings the enhanced and quality data in the field of medical field. In earlier technique of the medical field, such as MRI, CRI,X-rays, CT scan has been used and produced the result as low quality of image to detect the symptoms and diseases of the patients[8].

Using DWT the data's are formed as matrix for conversion process, which is used to soften the noise and its texture. The overall result and their behavior of the model has been estimated by their PSNR level and produces better results by comparing the previous models and produces the texture smoothness, and preserves the edges and removes unwanted noise present the images in the area of medical field[8].

This paper is organized as follows: a brief introduction of mammogram images in Section 1, review of different approaches presented in Section 2, the proposed system of de-noising of mammogram images is given in Section 3, and conclusion and future work are shown in Section 4.

\section{RELATED WORK}

In most of the review papers, different approaches have been given to DE-noise an noisy image based on some useful medical criteria. The ordinary noises present in the medical image are impulse noise salt and pepper noise, Gaussian noise etc. Based on these images, the real image that is input image given by the user looks blurred, and it is structure less and cannot be able to identify the exact images present in the medical report. This may lead to poor identification of image and cannot diagnosis the disease of the patients.[8]. These noises tend to blur the edges of the image, and it corrupts its originality present in the image and it does not sharpen and smoothen the image data and its performance [8]. Filtering techniques are used in order to improve the quality of the image. Based on the filtering technique used, unnecessary noises which are commonly available in the images has been removed. By evaluation result it shows the properties of image binarization which provides impulsive noise removal, piecewise smoothing, and sharp edge preservation of the image.[8].Through many algorithms such as DWT,FFT its performance can be estimated. Based on DWT and singular value decomposition, it decomposes the input image into four frequency sub bands and estimates it by low sub band images. It reconstructs the enhanced image by applying its inverse. The results are compared with conventional image equalization techniques such as brightness, preserving dynamic histogram equalization methods.[10].

Similarly techniques used for contrast enhancement on mammographic images broadly categories into six category such as unsharp masking, adaptive neighbourhood contrast enhancement histogram equalization, spatial filtering , wavelet enhancement and fuzzy model[14]. Among above mentioned techniques, adaptive contrast enhancement [15, 16] and wavelets $[17,18]$ proved to be more suitable for mammography. Adaptive contrast enhancement methods use a local contrast definition and then image is enhanced by improving the contrast measure via a contrast modification function [10]. For de-noising mammogram images wavelet filters such as sym8, coif1, haar,db3and db4 are used at certain level of hard and soft threshold functions. Different wavelet filters with obtained PSNR(Peak Signal-to-Noise Ratio) values for hard and soft threshold functions can be calculated for comparing the images. The concept of Wavelet Thresholding de-noising is completely based on the energy of the signal to be defined concentrates on some wavelet coefficients, whereas the energy of noise spreads throughout all wavelet coefficients. Similarity between the basic wavelet and the signal to be defined plays a very important role, making it possible for the signal to concentrate on fewer coefficients. The components of the impulse should be made as prominent as possible so as to improve the performance of impulse isolation. De-noising through the Wavelet threshold is a very efficient method, the aim of which is to eliminate independent and identically distributed Gaussian noise available in the images [19].

\section{PROPOSED SYSTEM}

In the proposed system, discrete wavelet transform algorithm is developed to remove the unwanted noises present in given mammogram image. There are fallowing modules in the proposed paper. The first module explains about resizing and filtering method. When the Mammogram image is given as input by the user, it is resized to $512 * 512$ enlarged pixel to process the data. The original image size is $256 * 256$ pixels which is given as input. Once the image is 
resized, the images are separated as rows and columns and then process it according to the brain image. Once the image pixel is resized, it is then filtered by using Gaussian filtering technique.

The main function of Gaussian filter is to minimize the low and high signals from distortion. The data's are checked based on the image resolution in filtering methodology A Gaussian filter is a filter whose impulse response have to be perform by the operation of Gaussian function. Gaussian filters have the properties such as that cannot be no longer without the overshoot of a step function input since reducing the rises and falls time[9].

These behaviors are very nearer to be connected with the facts that make the Gaussian filter to minimum the possible group delay. The Gaussian filter is non-causal that defines the filter window are symmetric in nature above the origin of the time-domain. These forms the Gaussian filter physically unrealizable and are usually of no consequence to the applications from where the filter bandwidth is much greater to the signal. In real-time systems, a delay incurs because incoming samples are needed to fill the filter window before the filter is to be applied to the signal [14].

Advantages of the Gaussian filter over the other known filters is that ,no amount of delay that helps to make a Gaussian filter causal, because the Gaussian functions are never zero. Secondly Focal element receives the heaviest weights which consist of having the highest Gaussian value that deals with the neighborhood elements receives the smaller weights of their distance with the focal element value increases[7].

Once the image is constricted through the Gaussian filter , it is passed through the other modules of proposed system and those modules for removing the noise and brings the best visual quality to the doctors to identify the diseases of the patient.

Based on the system architecture, the entire process is performed. There are four modules in this paper. Based on the modules, process gets estimated and evaluated. The raw Mammograms images generally contain noise, artifacts and are inconsistent to interpret. In order remove noise from mammogram noise reduction and Image Sharpening are performed. In pre-processing of the mammogram images Noise reduction and Image enhancement by sharpening and smoothing are performed.Reduction of noise from mammogram images involves existing artifacts like written labels , which is to be removed that can be possible with by cropping the images.

The process of pruning of images removes all possible background noise which is done by adding salt and pepper noise and then removing the noise using "Median Filtering". Image Sharpening Enhancing the high-frequency components of an image leads to an improvement in the visual quality. Mammogram images are passed through the Image filtering that is Gaussian Filter then resultant images processed by discrete wavelet transform. Final processed image of discrete wavelet transform are compared with different available techniques.

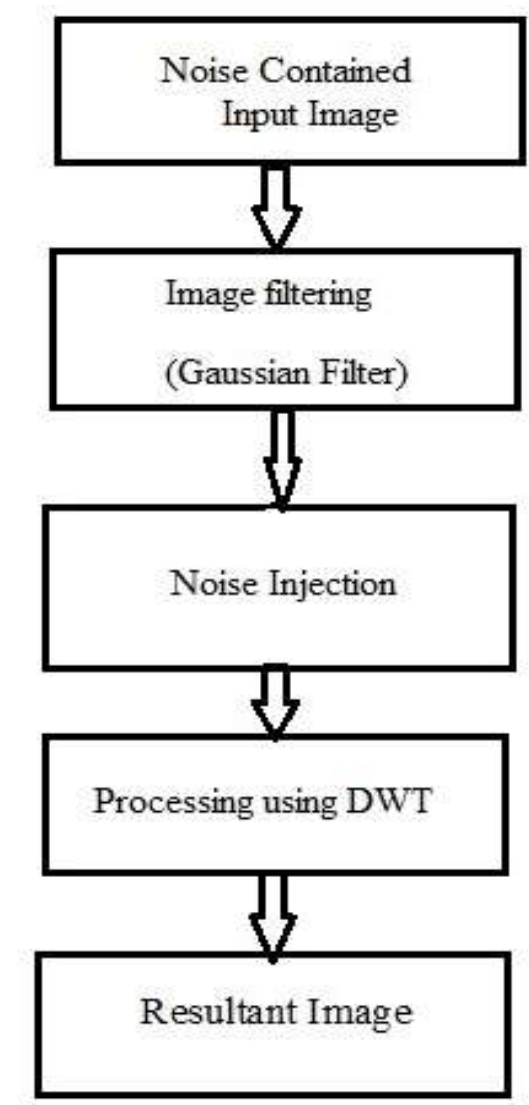

FIGURE. SYSTEM ARCHITECTURE OF PROPOSED WORK. 


\section{CONCLUSION AND FUTURE WORK}

This paper presents an algorithm for De noising mammogram images. Our algorithm will demonstrates better performance over existing available schemes. In image denoising technique, several methodologies have been developed and have been used to reduce the different types of noises present in the image. We have presented DWT methods for noise removal and the Gaussian filtering technique has been emerged in this paper. Based on these methods, the image quality will be improved as well as the doctors can easily identify the diseases based on the report and can rectify their problems. The issue The issue that we consider for future work, we will investigate other parameters in images to make images more clearly without losing any important data.

\section{REFERENCES}

[1] J. Portilla, V. Strela, M. J. Wainwright, and E. P. Simoncelli, "Image denoising using a scale mixture of Gaussians in the wavelet domain," IEEE Trans. Image Process., vol. 12, no. 11, pp. 1338-1351, Nov. 2003.

[2] Mónica Penedo, William A. Pearlman, Fellow, Pablo G. Tahoces, Member, Miguel Souto, and Juan J. Vidal,” Region-Based Wavelet Coding Methods for Digital Mammography", IEEE TRANSACTIONS ON MEDICAL IMAGING, VOL. 22, NO. 10, OCTOBER 2003.

[3] P. G. Tahoces, J. Correa, M. Souto, C. González, L. Gómez, and J. J. Vidal, "Enhancement of chest and breast radiographs by automatic spatial filtering," IEEE Trans. Med. Imaging, vol. 10, pp. 330-335, Sept. 1991.

[4] M. Souto, J. Correa, P. G. Tahoces, D. Tucker, K. S. Malagari, J. J. Vidal, and R. G. Fraser, "Enhancement of chest images by automatic adaptive spatial filtering,” J. Digital Imaging, vol. 5, pp. 223-229, Nov. 1992.

[5] Efficient techniques for color image noise reduction, The Research Bulletin of Jordan, Volume, 2,Mrs. C.Mythili

[6] ,Dr.V.K avitha, 2012.

[7] Noise cancellation on ECG and Heart rate signals using the undecimated wavelet transform, (IJERA) ISSN: 2248

[8] 9622, Vol. 1, Issue 4, pp. 1962-1970,2010

[9] María V. Sainz de Cea and Yongyi Yang,"Case-adaptive decision rule for detection of clustered microcalcifications in mammograms", IEEE 2015 .

[10] Nithya. K, Aruna.A, Anandakumar.H, Anuradha.B," A Survey On Image Denoising Methodology On Mammogram Images", INTERNATIONAL JOURNAL OF SCIENTIFIC \& TECHNOLOGY RESEARCH , ISSN 2277-8616,VOLUME 3, ISSUE 11, NOVEMBER 2014.

[11] "Edge preserving pre-filtering for document image binarization", National University of Singapore.

[12] Satellite Image Contrast Enhancement using DWT and singular value decomposition", IEEE GEOSCIENCE AND REMOTE SENSING LETTERS, VOL. 7, NO. 2, APRIL 2010.

[13] Enhancement and preprocessing of image using filtering,Roopashree.S, Sachin Saini, Rohan Ranjan Singh, International Journal of Engineering and Advanced Technology (IJEAT), ISSN: 2249 - 8958, Volume-1, Issue-5, June 2012.

[14] J. Méndez, P. G. Tahoces, M. J. Lado, M. Souto, and J. J. Vidal, "Computer-aided diagnosis: Automatic detection of malignant masses in digitized mammograms,” Med. Phys., vol. 25, pp. 957-964, June 1998.

[15] M. J. Lado, P. G. Tahoces, A. J. Méndez, M. Souto, and J. J. Vidal, "Evaluation of an automated wavelet-based system dedicated to the detection of clustered microcalcifications in digital mammograms," Med. Inform. Internet Med., vol. 26, pp. 149-163, July 2001.

[16] M. Adel , D. Zuwala+, M. Rasigni+ and S. Bourennane," Noise reduction on mammographic phantom images", Electronic Letters on Computer Vision and Image Analysis 5(4):64-74, 2006.

[17] A.P. Dhawan, G. Buelloni, R. Gordon, "Enhancement of mammographic features by optimal adaptative neighborhood image processing", IEEE Trans. Med. Imaging, 5(1):8-15, 1986.

[18] R. Gordon, R.M. Rangayyan, "Feature enhancement of film mammograms using fixed and adaptative neighbourhoods", Applied Optics, 23(4):560-564, 1984

[19] A.F. Laine, S. Schuler, J. Fan, W. Huda, "Mammographic feature enhancement by multiscale analysis", IEEE Trans. Med. Imaging 13(4), 725$740,1994$.

[20] A.F. Laine, J. Fan, W. Yang, "Wavelets for contrast enhancement of digital mammography”,IEEE Eng. Med. Biol. Magazine, 14:536-550, 1995.

[21] Saima Anwar Lashari, Rosziati Ibrahim, Norhalina Senan,” De-noising Analysis of Mammogram Images in the Wavelet Domain using Hard and Soft Thresholding".

[22] Saima Anwar Lashari, Rosziati Ibrahim, Norhalina Senan,” Effect of Presence/Absence of Noise in Mammogram Images Using Fuzzy Soft Set Based Classification”, ISBN: 978-1-4799-6211-2, IEEE, 2015. 